\title{
III.
}

\section{Über die Schleimzone des menschlichen Magen- und Darmepithels vor und nach der Geburt.}

(Aus dem Pathologischen Institut zu Berlin.)

\author{
Von \\ Else von der Leyen.
}

Herr Geheimrat Orth beauftragte mich im Oktober 1903 , im Anschluß an die Veröffentlichung ron Disse vom Januar 1903 „Über die Durchgängigkeit der jugendlichen Magendarmwand für Tuberkelbazillen " ${ }^{1}$ die Schleimdecke des Magen- und Darmepithels von Kindern in den ersten Lebenstagen zu untersuchen. Während ich damit beschäftigt war, veröffentlichte Geheimrat v. Behring im April 1904 eine Anzahl von Disse redigierter Sätze, in denen die früher erhobenen Befunde ${ }^{2}$ erweitert werden. Ferner wies P. Reyher ${ }^{3}$ in einer im Juli 1904 erschienenen Arbeit an der Hand von vorzüglichem Material die von Disse gemachten Schlußfolgerungen gänzlich zurück. Reyher hat auch die bisher erschienene Literatur zusammengestellt, was bis dahin nicht geschehen war. Da es sehr schwierig ist, menschliche Magenschleimhaut mit gut erhaltenem Epithel zu bekommen und daher nur eine verhältnismäßig kleine Anzahl von Untersuchungen an dem Magenepithel junger Kinder vorgenommen ist, halte ich es nicht für überflüssig, auch meine Fälle zu veröffentlichen. Dabei erwähne ich die bisher gemachten Untersuchungen nur, soweit es zur Einleitung notwendig ist, und verweise im übrigen auf die bei P. Reyher zusammengestellte ausführliche Literaturübersicht.

Im November 1902 hielt v. Behring ${ }^{4}$ einen Vortrag „Über Zustandekommen und Bekämpfung der Rindertuberkulose", in dem er angab, daß der Intestinaltraktus (worunter man gemeinhin Magen- und Darmkanal versteht) Neugeborener, weil eine kontinuierliche Sehleimschicht fehle, für viele Substanzen durchgängig sei, die späterhin nur noch nach Verletzung der 
Schleimhaut resorbiert wurden. Er berief sich auf die Untersuchungen Disses ${ }^{1}$, die ausführlich im Januar 1903 veröffentlicht wurden.

Disse beschreibt bei dieser Gelegenheit:

1. die Magenschleimhaut eines menschlichen Embryo im letzten Monat,

2. die eines neugeborenen Meerschweinchens,

3. die eines 24 Std. post partum getöteten Hundes.

Die Gewebsteile müssen, nach den Abbildungen zu urteilen, sehr bald nach dem Tode in die Konservierungsflüssigkeit gekommen sein. Er härtete in Hermannscher Flüssigkeit, färbte nach eigens von ihm angegebener Methode auf Schleim. Er fand, „daß die Schleimdecke beim älteren Foetus wie beim Neugeborenen nicht nur viel dünner wie beim Erwachsenen ist, sie ist auch unterbrochen und besteht aus vielen Stückchen, die durch Zellprotoplasma voneinander getrennt werden."

„Eine Zerlegung der Zellen in zwei Zonen, wie beim Erwachsenen, ist nicht durchführbar; die Zelle erscheint einheitlich, aber jede Zelle enthält nahe der freien Fläche eine rundliche ... Einlagerung ... wir ... wollen sie als Schleimpfropf bezeichnen."

„Im Laufe des ersten Lebenstages verschwindet das protoplasmatische Netzwerk, das die einzelnen Schleimpfröpfe voneinander trennt. ... Dadurch wird eine zusammenhängende Schleimdecke gebildet, die zwar viel dünner ist als beim Erwachsenen, die aber das Zellprotoplasma durchweg vom Lumen des Magens trennt.... Es ist auch wahrscheinlich, daß bei größeren Tieren die normale Dicke der Schleimdecke später erreicht wird als bei kleineren." Ferner: "Die Darmschleimhaut neugeborener Tiere dagegen gleicht schon der des Erwachsenen." Sonst ist vom Darm nicht die Rede. Dagegen findet sich später die Stelle: "Wir sehen in der raschen Ausbildung einer zusammenhängenden Schleimschicht eine gleichzeitig mit dem Beginn der Nahrungsaufnahme auftretende Schutzvorrichtung des Organismus, die zur Sicherung gegen das Eindringen schädigender Potenzen vom Darmkanal her dient."

In der v. Behring schen Veröffentlichung vom April ${ }^{2}$ steht: 
„2. Bei Säugetieren mit einfachem Magen ist zur Zeit der Geburt die Schleimlage unterbrochen durch Protoplasmagitter, und die einzelnen ... Partien der vorhandenen Schleimlage sind bei neugeborenen Individuen viel dünner als beim Erwachsenen."

3. „Die Zeit, die nach der Geburt bis zur vollständigen Ausbildung der Schleimlage verstreicht, ist nicht nur bei verschiedenen Tierarten, sondern auch bei einzelnen Individuen derselben Art verschieden, für den Menschen konnte sie bis jetzt aus Mangel an ausreichendem Material noch nicht genau bestimmt werden."

.4. Beim neugeborenen Menschen ... findet man schleimarme und schleimreichere Bezirke nebeneinander. Daher ist für eine genügende Orientierung über die Schleimdecke des Magens die Untersuchung vieler größerer Schleimhautstücke an Schnittreihen eine unabweisliche Forderung."

„8. In allen menschlichen Magen von Ëmbryonen und Neugeborenen, die bisher von mir untersucht worden sind, war also die Schleimdecke des Epithels eine unterbrochene, trotz bedeutender Schleimproduktion einzelner Stellen."

"Ich konnte bisher nicht mit Sicherheit feststellen, ob der Darmtraktus zur Zeit der Geburt sich so verhält wie später, oder ob Unterschiede im anatomischen Verhalten bestehen. Insbesondere über den Blinddarm besitze ich keine eigenen Erfahrungen." Trotz dieser Worte und obgleich ausschließlich vereinzelte Magenuntersuchungen, absolut keine Darmbefunde beschrieben wurden, ist auch hier wieder von der "Schutzlosigkeit der intestinalen Schleimhäute gegen Tuberkelbazillen" bei Neugeborenen die Rede.

Soweit Disse und v. Behring.

Nach Kölliker ${ }^{5}$ kann man an jeder Magenepithelzelle in frischem Zustande zwei Abteilungen unterscheiden: eine tiefe, der Schleimhaut aufsitzende, welche den länglichen Kern enthält, eine oberflächliche, welche etwas dunkler erscheint. Die beiden Abteilungen gehen am ganz frischen Objekt ohne scharfe Grenze ineinander über. Die Ausdehnung der dunkleren Abteilung kann bald größer, bald geringer sein. Die quellbare (obere) Substanz der Cylinderzellen des Magens enthält Schleim, 
da sie mit den spezifischen Schleimfärbemitteln sich färben läßt, freilich nicht so intensiv, wie die Zellen der Schleimdrüsen und Becherzellen. ... Die verschiedenen, durch Reagenzien erzeugten Bilder, namentlich die größere oder geringere Ausdehnung des anscheinend entleerten, aber mit einer hyalinen, pfropfartigen Masse erfüllten Zellanteils hängt von der Menge und dem Maße der Reife der Sekretstoffe ab."

Das bezieht sich auf den Magen von Erwachsenen. Kindermagen wurden von Werber ${ }^{6}$, Toldt $^{7}$, Baginsky ${ }^{8}$, Fischl ${ }^{9}$, Schmidt ${ }^{10}$, Coudereau ${ }^{11}$, Kalopothakès ${ }^{12}$, Bloch ${ }^{13}$, de Lange ${ }^{14}$, Disse ${ }^{1}$, Benda ${ }^{15}$, Reyher ${ }^{3}$, Tierfoeten wurden von Sacerdotti ${ }^{17}$ and Disse ${ }^{1}$ untersucht. $^{1}$ )

Coudereau ${ }^{11}$ fand, daß beim menschlichen Foetus vom 5. Monat die Drüsen mit becherzellenartigem Epithel bedeckt sind, das Schleim sezerniert. Schmidt ${ }^{10}$ fand beim $5 \frac{1}{2}$ monatlichen menschlichen Embryo eine zusammenhängende Schleimschicht, Benda ${ }^{15}$ beim 8 monatlichen, Sacerdotti ${ }^{16}$ ebenso beim $14 \mathrm{~cm}$ langen Rindsfoetus.

Auf die Arbeit von P. Reyher möchte ich etwas ausführlicher eingehen. Er untersuchte die zum größten Teil lebenswarm eingelegte Magenschleimhaut von 9 menschlichen Individuen verschiedenen Lebensalters: 1 Foetus von 7 Monaten, 1 Embryo von 8 Monaten, 5 neugeborene Kinder, 114 Tage altes, 110 Tage altes Kind, 1 Erwachsenen. Er konservierte in Orthscher Lösung, härtete mit Alkohol und bettete in Paraffin ein. Er färbte teils mit den bekannten Schleimfärbemitteln, Mucikarmin, Muchämaein, Thionin, teils mit van Gieson oder mit dem Ehrlich-Biondi-Heidenhainschen Dreifarbengemisch. So erzielte er zwar keine distinkte und spezifische Schleimfärbung, aber die einzelnen Zellabschnitte waren durch Abstufung der Farbentöne deutlich von-

1) Am 19. Januar 1905 hat im ärztlichen Vereine zu Marburg Asch off ein Referat gehalten: „Zur Histologie der Darmschleimhant der Neugeborenen“. Er kommt zu dem gleichen Resultat wie wir, „daß in der mit den bisherigen Methoden nachweisbaren histologischen Zusammensetzung des Oberflächen- und Drüsenepithels des Magens und Darmes beim Neugeborenen eine Erklärung für die leichtere Durchlässigkeit gegen Bakterien nicht gegeben ist“. 
einander zu unterscheiden. P. Reyher fand in sämtlichen Fällen einen ununterbrochenen Schleimbelag des in allen Fällen gut erhaltenen Magenepithels. Die Untersuchung einer verhältnismäßig so großen Reihe gut konservierter Präparate von Magenschleimhant neugeborener Kinder und Embryonen ist vor Reyher überhaupt noch nicht veröffentlicht worden.

Ich habe im ganzen 13 Fälle untersucht. Brauchbar davon sind nur 6 Fälle, bei denen das Epithel kontinuierlich erhalten war; die anderen 7 Fälle zeigten, obgleich alle 13 in gleicher Weise vorbehandelt waren, nur streckenweise gut erhaltenes Epithel. Tch führe sie hier mit an, weil das Epithel, soweit es vorhanden war, den gleichen Anblick bot, wie die 6 gut erhaltenen! Fälle.

Ich untersuchte den Magen eines Foetus von $27 \mathrm{~cm}$ Länge, 9 Magen von neugeborenen Kindern, 1 Magen eines Kindes, das 9 Stunden gelebt hatte, eines, das 2 Tage, eines, das 14 Tage gelebt hatte, eines, das etwa 1 Jahr alt geworden war.

Der Magen des Foetus kam etwa 16 Stunden, nachdem der Abort erfolgt war, in die Konservierungsflüssigkeit. Sieben Kindern wurde möglichst bald nach dem Tode entweder $10 \%$ Formalin in die Bauchhöhle gespritzt, oder es wurde mittels eines Nelaton-Katheters Formalin in den Magen per os eingeführt. 5 Fälle wurden möglichst bald post mortem seziert; ein Teil des Magens wurde in 10\% Formalin, ein anderer in ein Gemisch von Sublimat, Chromsäure, Eisessig gelegt. Es gelangten in jedem Fall verschiedene Teile des Magens zur Untersuchung. In allen Fällen, wo ich Formalin in die Bauchhöhle injiciert habe, wurden Teile des Dünn- und Dickdarms eingelegt und untersucht. Konserviert wurde in Formalin, in 5 Fällen in Sublimat-Chromsäure-Eisessigmischung, da ich die Dissesche Färbung versuchen wollte, gehärtet in Alkohol, in Paraffin eingebettet. Die untersuchten Schnitte waren meist 10 u dick, einzelne Male dünner.

Gefärbt wurde mit Hämatoxylin-Eosin, mit van Gieson. Außerdem wurden sämtliche mir bekannten Schleimfärbemethoden durchprobiert. Gute Resultate erhielt ich nur mit Thionin und Toluidinblau, die Dissesche Färbung ist mir, nicht gelungen. Da ich genau nach Vorschrift färbte, kann 
ich die Ursache des Mißlingens nur in der durch ein Versehen etwas verschiedenen Konservierungsmethode [ $\mathrm{HgCl}_{2}$ statt $\mathrm{AgNO}_{3}$ ] suchen. Toluidinblau färbte sowohl bei den in Sublimat, wie bei den in Formalin eingelegten Gewebsteilen, bei letzteren intensiver und dunkler.

Mit Thionin gefärbte Präparate zeigten, in Wasser betrachtet, eine blaurote Färbung des oberen Endes der Epithelzellen; durch Alkohol wurde die Farbe sofort ausgezogen. Färbt man die Schnitte mindestens 24 Stunden in einer verdünnten, wässerigen Lösung von Toluidinblau, entfärbt in verdünntem Alkohol, hellt mit Bergamottöl (nicht mit Xylol) anf und bettet in Kanadabalsam ein, so färbt die Schleimschicht der Epithelzellen sich dunkelrotriolett bis beinahe schwarz, die Zellkerne und das übrige Gewebe werden blau, die Mastzellen rotviolett. Die Färbung ist nicht immer gleichmäßig intensiv beim Magenschleim. In zwei Fällen vermochte ich ohne mir ersichtlichen Grund nur leichte Anfärbung zu erzielen. Die Becherzellen des Darms färbten sich ausnahmslos prachtvoll schwarzviolett. Man kann Reyher nur Recht geben, wenn er -sagt, daß eine spezifische Schleimfärbung nicht notwendig ist. Sie ist natürlich instruktiver und hübsch anzusehen. Aber man unterscheidet an jeder Epithelzelle einen unteren Abschnitt mit Kern und einen oberen überall deutlich und scharf abgegrenzten Teil, der etwas aufgequollen erscheint und mit den gewöhnlichen Färbemethoden beinahe farblos bleibt. Das ist der Schleimabschnitt der Zelle - Sache einer weiteren Untersuchung wäre es, die Ursache des wechselnden Verhaltens des Magenschleims Schleimfarbstoffen gegenüber zu ergründen.

Bei den untersuchten Darmteilen, die ich hier nur erwähnen will, war das Epithel in allen Fällen vorzüglich konserviert. Die Becherzellen, ebenso zahlreich wie bei älteren Kindern, färbten sich mitToluidinblau intensiv schwarzblan. Im Dickdarm, manchmal auch im Dünndarm, waren die einzelnen Becherzellen durch einen strichförmigen, sehwarzen Schleimbelag miteinander verbunden. Im Blinddarm fanden sich bei dem $27 \mathrm{~cm}$ langen Foetus noch keine deutlich färbbaren Becherzellen, dagegen bei den Neugeborenen ebenso zahlreiche und gut ausgebildete wie bei älteren Kindern. Der Befund wàr in allen Fällen ganz gleichmäßig. 
Es folgt die Aufzählung meiner Fälle:

1. $27 \mathrm{~cm}$ langer Foetus. 18 Stunden p. abort. seziert. Magen $2 \frac{1}{2} \mathrm{~cm}$ lang, ganz mit Schleim gefüllt. Kleiner Teil des Fundus in Chromsäure-Sublimat-Essigsäuregemisch, der übrige Magen in $10 \%$ Formalin eingelegt. Färbung mit Hämatoxylin-Eosin-Orange und Toluidinblau. Es wurden sehr zahlreiche Schnitte verschiedener Teile und kleine Schnittserien untersucht.

Das Epithel ist durchweg gut erhalten. Es sind meist ungeteilte, einfache Schlauchdrüsen. Die Submucosa ist ziemlich breit. An den Epithelzellen unterscheidet man durchweg zwei Abschnitte; einen unteren; etwa die Hälfte der Zelle einnehmenden, mit mattblau (To.) gefürbtem Protoplasma und rundem, manchmal. oben-, manchmal untenliegendem Kern, einen oberen, etwas gequollenen, deutlich abgegrenzten Abschnitt, der sich hellrotviolett färbt. Dazwisehen finden sich ganz vereinzelte Zellen, die wie die Becherzellen des Darms aussehen und sich dunkler, etwa wie die Darmbecherzellen, färben. Diese hellrotviolette Schicht bedeckt kontinuierlich die ganze Oberfläche des Magens.

Der Mageninhalt besteht aus Schleim, Gerinnsel und Zellen.

2. Neugeborenes Kind (Fall 119, 1904). Kurz post mortem. (etwa 12 Stunden) seziert, Teile des Magens in Chromsäure-Sublimat-Eisessiggemisch, andere Teile in 10\% Formalin eingelegt.

Epithel zum größten Teil gut erhalten, Kerne rund, in der Mitte der Zelle. Das obere Drittel bis die Hälfte der Zelle einnehmend scharf konturierte, schwarzviolett gefärbte, überall kontinuierliche Schleimschicht. Schmale Mucosa, schmale, tubulöse Drüsen. Mastzellen im submucösen Gewebe.

3. Neugeborenes Kind, mit Formalin injiciert, 1904. Es gelangten Abschnitte von der Fundus- und Pylorusgegend zur Untersuchung.

Sehr gut im Zusammenhang erhaltenes, nur manchmal ètwas abgehobenes Epithel. Man sieht wieder einen oberen, scharf abgegrenzten Zellabschnitt, $\frac{1}{6}$ bis $\frac{1}{3}$ der Zelle einnehmend, der den ganzen Magen kontinuierlich überzieht. Er färbt sich matt mit Toluidinblau; dagegen befinden sich auf dem Epithel reichlich schwarze, fädige Auflagerungen. Die Drïsenlumina sind z. T. mit solchen schwarzen, fädigen Massen gefiullt.

Außerordentlich zahlreiche Mastzellen, sowohl in der Submucosa wie in dem Bindegewebe der Magenleistchen.

4. 14 Tage altes, an Gastroenteritis gestorbenes Kind (211, 1904) Sublimat-Chromsäure-Eisessiggemisch.

Mucosa breit. Epithel zum Teil gut erhalten. Ausgedehnte, granblau gefärbte Zone, $\frac{1}{3}$ bis $\frac{2}{3}$ des oberen Zellendes einnehmend, kontinuierlich. Kern im untersten Drittel der Zelle, oft dicht an die Schleimzone anstoßend.

5. Magen von Neugeborenem $(1041,1903)$, per os mit $10 \%$ Formalin gefüllt. Epithel der Magendrüsen fast überall gut erhalten. $\frac{1}{2}$ bis $\frac{2}{3}$ des oberen Zellteils wird von einer körnig schwarzblau gefärbten 
Masse eingenommen, die ununterbrochen ist, in der Dicke etwas wechselt. Auf der Epithelschicht an manchen Stellen schwarz gefärbte Massen.

6. Magen von Neugeborenem $(1042,1903)$, mit $10 \%$ Formalin per os gefüllt.

Epithel gut erhalten. Kerne teils an Zellgrunde, teils in der Mitte der Zellen. $\frac{1}{3}$ bis $\frac{2}{3}$ der Zelle einnehmend, manchmal dicht an den Kern stoßend, manchmal etwas über ihm liegend, intensiv schwarzblau gefärbte, zusammenhängende Zone. Die Mucosa ist sehr schmal, die Drüsen stehen nicht an allen Stellen dicht, sondern sind durch Bindegewebe voneinander getrennt. Die Drüsen sind kurz, tubulös, wenig verzweigt. Auffallend ist die verschiedene Dicke des Schleimbelags an dicht nebeneinanderliegenden Schleimhautpartieen. In der Submucosa wenige Mastzellen. Die Drüsenlumina sind z. T. mit blauschwarzen Massen gefüllt.

Die übrigen Fälle -1 Kind von 9 Stunden, 1 von 2 Tagen, 1 von 1 Jahr, 4 Neugeborene - zeigten an den Stellen, wo das Epithel erhalten war, den gleichen kontinuierlichen, graublau bis schwarzblau gefärbten Schleimbelag. Dieser Belag war bei dem einjährigen Kinde ebenso breit wie beim Neugeborenen.

Hieraus geht wieder, im Gegensatz zu Disses Untersuchungen und v. Behrings auf sie gestützten Schlußfolgerungen und in Übereinstimmung mit allen sonst vorangegangenen Forschungen hervor, daß der obere Teil des Magenepithels vom Foetus von $27 \mathrm{~cm}$ ab bis $\mathrm{zu}$ dem rom einjährigen Kinde von einer kontinuierlichen, meistens distinkt färbbaren Schleimzone gebildet wird. Diese Schleimzone ist von wechselnder Breite; verschieden breit bei gleichalterigen Kindern (159, 1042), erscheint sie gerade bei dem Foetus von $27 \mathrm{~cm}$ auffallend hoch. Der Darmkanal des Foetus und der neugeborenen Kinder zeigt in bezug auf Entwicklung und Zahl der Becherzellen einen von dem älterer Kinder in keiner Weise abweichenden Befund.

Zum Schluß sage ich Herrn Geheimrat Orth für die Uberlassung dieser Arbeit, und den Herren von der geburtshilflichen Klinik und vom Pathologischen Institat, die mir zur Erlangung des Materials behilflich waren, meinen besten Dank.

\section{Literatur.}

1. Disse, Untersuchungen über die Durchgängigkeit der jugendlichen Magen- und Darmwand für Tuberkelbazillen. Berlin. klin. Wochenschrift Nr. 41, 1903, S. 4. 
2. Tuberkuloseentstehıng, Tuberkulosebekämpfung und Säuglingsernährung. E. v. Behring, Beiträge zur experimentellen Therapie, Heft 8, Hirschwald, 1904.

3. Dr. Paul Reyher, Über die Ausdehnung der Schleimbildung in den Magenepithelien des Menschen vor und nach der Geburt. Jahrbuch f. Kinderheilkunde 60 , der dritten Folge 10. Bd., Heft 1, 1. Juli 1904, Norwegen-Berlin, S. 16 ff.

4. von Behring, Zustandekommen und Bekämpfung der Rindertuberkulose. Berlin. tierärztl. Wochenschr. 1902, Nr. 47.

5. Kölliker, Handbuch d. Gewebelehre. Bearb. v. V. Ebner. Leipzig 1902, Bd. III.

6. Werber, A., Beiträge zur pathologischen Anatomie des pädatrophischen Darms mit Bemerkungen zum Bau des normalen Darms bei Neugeborenen. Bericht über die Verhandlungen der Naturforscher-Gesellsch, in Freiburg i. Br., Bd. III, 1865.

7. Toldt, C., Die Entwicklung und Ausbildung der Drüsen des Magens. Sitzungsberichte der Kaiserlichen Akademie der Wissenschaften, math.-naturw. Klasse, Bd. 82, Hft. 1 u. 2, 1880, S. 57.

8. Baginsky, Untersuchungen über den Magendarmkanal des menschlichen Kindes. Dieses Archiv Bd. 89, 1882.

9. Dr. Rudolf Fischl, Beiträge zur normalen und pathologischen Histologie des Säuglingsmagens. Zeitschr. f. Heilkunde Bd. XII, 1891, S. 395 .

10. A. Schmidt, Untersuchungen über das menschliche Magenepithel nnter normalen und pathologischen Verhältnissen. Dieses Archiv Bd. 143, 1896, S. 66 .

11. Dr. A. Coudereau, Structure des glandes de l'estomac. Travaux du laboratoire de physiol. et de la faculté de médecine de Paris, I vol., Paris 1885 , p. $19-29$.

12. Maria Kalopothakès, Contributions à l'étude de la structure normale de l'estomac chez le foetus et le nouveau-né. Société anatom. de Paris 1894, p. 685-696.

13. Bloch, Untersuchungen über den Magendarmkanal des Säuglings. Jahrbuch für Kinderheilkunde Bd. 58, 1903.

14. Cornelia de Lange, Zar normalen und pathologischen Histologie des Magendarmkanals beim Kinde. Archiv f. Kinderheilkunde Bd. 51, Leipzig, Teubner, 1900.

1.5. Benda, Diskussion in der Berliner mediz. Gesellschaft. Berlin. klin. Wochenschr. 1904, Nr. 9, S. 232.

16. Sacerdotti, Uber die Entwicklung der Schleimzellen des Magendarmkanals. Monatsschr. f. Anatomie u. Physiologie Bd. 11, 1894, S. 501.

17. Sacerdotti, Sulla regenerazione dell' epitelio muciparo del tubo gastroenterico degl' anfibi. Atti dell' accademia di Firenze e Torino, Bd. 31, 1895/96, S. 870-881. 